was developed. The needs assessment identified three areas for targeted outreach: brothel services for heterosexual men, sauna for MSM and youth offender services. A health advisor outreach clinic was established and staffed 1-4 times a month. Outcome data from the clinics are presented.

Results The youth offender service clinic was the most successful outreach with 32 patients seen over 17 sessions (23 males and nine females). There were 15 males tested in the sauna over 18 sessions and four female sex workers tested in two clinic sessions. STIs were identified in $20 \%$ of all patients seen (two cases of Gonorrhoea, six cases of Chlamydia, two cases of syphilis). The mean percentage of patients seen in the outreach clinics who had never been to the GUM clinic was $71 \%$ (75\% in brothel, $84 \%$ in YOS, 34\% from sauna).

Discussion The targeted outreach has revealed a high level of STIs $(20 \%)$ in the target group as assumed. The targeted outreach service should reduce the rate of STIs by appropriate treatment, partner notification and counselling. Recording of testing has missed out on the recording of educational activities and other health promotion carried out at sessions. Targeted outreach will help to improve access to those who don't normally attend, improve patient care and help reduce STIs in the community.

\section{P152 IS IT FEASIBLE TO OFFER A CHLAMYDIA TEST OF CURE AT THE SAME TIME AS ROUTINE GONORRHOEA TEST OF CURE IN PATIENTS WITH BOTH INFECTIONS?}

doi:10.1136/sextrans-2012-050601c.152

H Colver, ${ }^{*}$ A Palfreeman. Leicester Royal Infirmary, Leicester, UK

Background BASHH guidelines recommend routine test of cure (TOC) for gonorrhoea by nucleic acid amplification technique (NAAT) 2 weeks after treatment if asymptomatic, while it is advised to defer chlamydia TOC until 6 weeks after treatment initiation. Chlamydia TOC is recommended only during pregnancy or if there is clinical suspicion of ongoing or re-infection, although there have been recent concerns about the efficacy of single dose azithromycin therapy. Many patients are co-infected with gonorrhoea and chlamydia, and with dual-platform NAATs it is simple and cost-effective to obtain chlamydia and gonorrhoea results from the same sample.

Aim To identify whether it is feasible to perform chlamydia and gonorrhoea NAAT TOCs simultaneously within 42 days of treatment in dual-infected patients.

Methods 38 patients with chlamydia who had repeat NAAT tests within 42 days of initiation of treatment were identified using clinical coding and pathology results. Demographic details, treatment type and time elapsed from initiation of treatment to TOC were obtained from patient notes.

Results $36 / 38(94.7 \%)$ of chlamydia TOCs taken 41 days or less since the initiation of treatment were negative, including $8 / 8(100 \%)$ of tests taken 14 days or less since chlamydia treatment. One positive test was from a male, the other from a female, 21 and 17 days after treatment respectively. Both were treated with azithromycin. The male patient denied sexual contact since treatment, and it was unclear whether the female patient was at risk of re-infection.

Conclusions $95 \%$ of patients in a clinical setting tested negative for chlamydia within 42 days of treatment. Performing a NAAT TOC for chlamydia and gonorrhoea simultaneously in dual-infected patients is therefore feasible, and is a cost-effective and convenient way to reassure patients that both infections have been eradicated. More work is needed to establish the cause of ongoing chlamydia positivity within 42 days of treatment.

\section{P153 \\ FEMALE GENITAL MUTILATION (FGM)-PROVIDING A HOLISTIC APPROACH AND CHALLENGING TABOOS IN A SEXUAL HEALTH SETTING}

doi:10.1136/sextrans-2012-050601c.153

L Dominguez, * L Dominguez, K Hopkins, 0 Mutalak, S Harish, A Nalabanda, S Osman, L Robinson, C Cohen, R Jones. Chelsea and Westminster NHS Foundation Trust, London, UK

Background In England and Wales nearly 66000 women are living with FGM and a potential 22000 girls are at risk each year. Due to increasing demand, a dedicated FGM service within the sexual health setting was initiated in partnership with a dynamic Somali facilitator and a voluntary community organisation.

Aims To analyse the attendances of women with FGM to our dedicated clinic.

Methods Retrospective analysis of all attendees to the FGM clinic between 1 December 2010 and 31 December 2011.

Results Of 197 attendees, 96\% were from Somalia (190); mean age was 38 (14-72 years) with $52 \%$ living locally. Of those in whom we had documented information, FGM was mainly undertaken for cultural reasons $(83 / 105,79 \%)$, the majority $(50 / 68,73 \%)$ being cut in groups at their home $(66 / 113,58 \%)$. Mothers were the main instigators (58/81, 71\%). Nearly half (84/173, 49\%) had female children and seven women reported having their daughters cut abroad. The majority were against the practice (112/114, 98\%). Reasons for attendance included chronic pelvic pain (57\%), dyspareunia (44\%) and sub fertility (24\%). There was a high uptake of sexual health screening, with almost all patients (191, 97\%) having serology for HIV and syphilis. Interestingly, no HIV was detected but $7 \%(13 / 185)$ were hepatitis B surface antigen positive and $31 \%$ $(58 / 185)$ had cleared hepatitis B. There were six diagnoses of late latent syphilis and two of chlamydia.

Conclusions Our Somali facilitator has played a key role in challenging the taboo of sexual health issues within FGM-practising communities, providing a service that is culturally and linguistically appropriate. The rate of blood-borne viruses in this vulnerable group proves the importance of engaging them with sexual health services. Fast track referrals to on-site gynaecology and hepatitis clinic have facilitated re-engagement of some patients with medical care.

\section{P154 WHO IS MOST AFFECTED BY STIGMA OF AN INTEGRATED SERVICE?}

doi:10.1136/sextrans-2012-050601c.154

${ }^{1} \mathrm{U}$ Sauer, ${ }^{2} \mathrm{~A}$ Singh, ${ }^{3} \mathrm{R}$ Pittrof. ${ }^{*}$ Barnet, Enfield and Haringey Mental Health Trust,

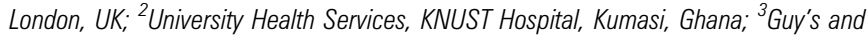
St. Thomas' NHS Foundation Trust, London, UK

Background Stigma of sexual health services creates barriers to access to care but it is not known if it affects all groups service users equally. Targeted stigma reduction requires this information.

Objective To determine which user groups of a level 3 One Stop Shop (OSS) sexual health services perceives the service as stigmatised.

Method Application of a validated quantitative tool to assess stigma among 200 unselected patients attending a OSS in outer London. We defined that the service was perceived as stigmatised if at least one moderately positive response indicating stigma was given.

Results The service was perceived as stigmatised by 55\% (111/200) of patients surveyed. Stigma of the service was more felt by men $(25 / 3964 \%)$ than by women $(84 / 160 ; 53 \%)$ and or those who classified themselves UK black (29/46; 63\%) rather than UK white $(19 / 66 ; 57 \%)$. Young men (age $<26$ years) $(12 / 20 ; 60 \%)$ perceived the service as less stigmatised than older men (13/19; 68\%) while 